\title{
PERSPECTIVE GROUND-BASED METHOD FOR DIAGNOSTICS OF THE LOWER IONOSPHERE AND THE NEUTRAL ATMOSPHERE
}

\author{
N. V. Bakhmetieva, G. I. Grigoriev and A. V. Tolmacheva \\ Radiophysical Research Institute (RRI), B. Pecherskaya str., 25/12a, \\ Nizhny Novgorod, 603950, Russia; nv_bakhm@nirfi.sci-nnov.ru
}

Received: 2012 November 13; accepted: 2012 November 28

\begin{abstract}
We present a new perspective ground-based method for diagnostics of the ionosphere and atmosphere parameters. The method uses one of the numerous physical phenomena observed in the ionosphere illuminated by highpower radio waves. It is a generation of the artificial periodic irregularities (APIs) in the ionospheric plasma. The APIs were found while studying the effects of ionospheric high-power HF modification. It was established that the APIs are formed by a standing wave that occurs due to interference between the upwardly radiated radio wave and its reflection off the ionosphere. The API studies are based upon observation of the Bragg backscatter of the pulsed probe radio wave from the artificial periodic structure. Bragg backscatter occurs if the spatial period of the irregularities is equal to half a wavelength of the probe signal. The API techniques makes it possible to obtain the following information: the profiles of electron density from the lower D-region up to the maximum of the F-layer; the irregular structure of the ionosphere including split of the regular E-layer, the sporadic layers; the vertical velocities in the D- and E-regions of the ionosphere; the turbulent velocities, turbulent diffusion coefficients and the turbopause altitude; the neutral temperatures and densities at the E-region altitudes; the parameters of the internal gravity waves and their spectral characteristics; the relative concentration of negative oxygen ions in the D-region. Some new results obtained by the API technique are discussed .
\end{abstract}

Key words: instrumentation: detectors - ionosphere: structure

\section{INTRODUCTION}

We present a short review of possibilities of the effective method for measurement of the ionosphere characteristics, based on a creation of the artificial periodic irregularities (APIs) by a radiation from a powerful SURA heating facility.

The technique based on artificial periodic irregularities (APIs) in the ionospheric plasma is one of the accredited methods in the ionosphere studies. This technique is based on (1) the action on the ionosphere by a powerful standing wave for creation of the periodic structures in electron density, (2) the sounding of APIs by short radio pulses and the registration of amplitudes and phases of the back-scattered signals to investigate their altitude-time dependence. Finally, some parameters of the ionospheric plasma and the atmosphere can be derived from the 


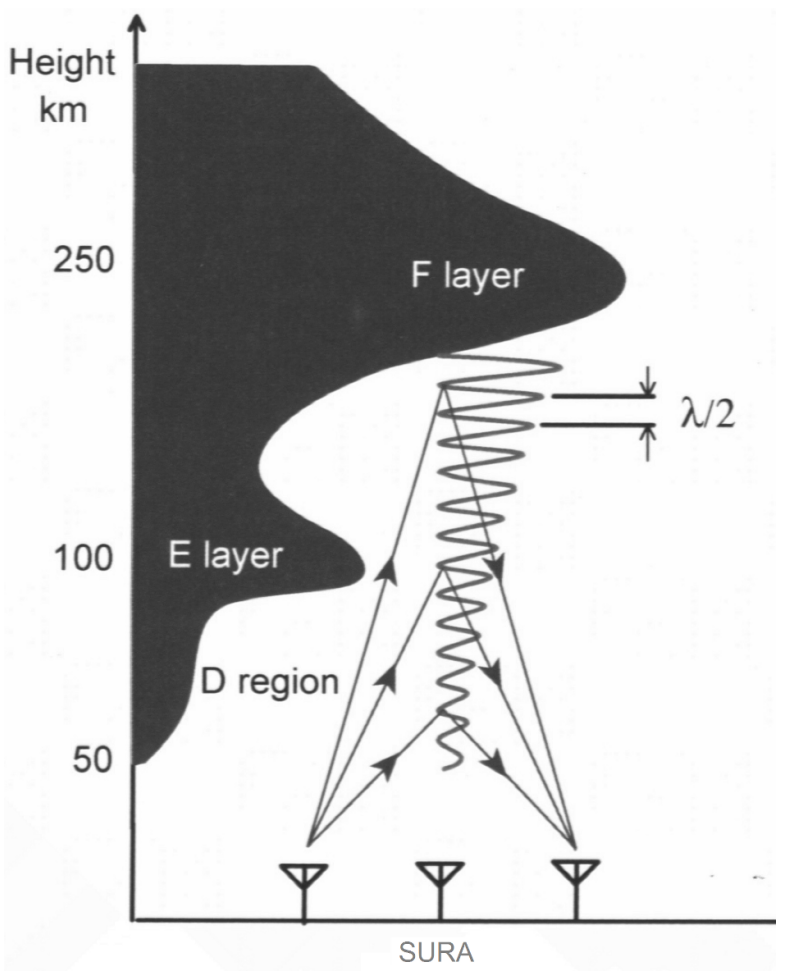

Fig. 1. A scheme for production of the artificial periodic irregularities of the ionospheric plasma and their sounding by pulsed signals.

data. During last years, the unique data on variations of the important parameters of the ionospheric plasma and the atmosphere have been received. Such parameters are the electron density, the vertical motion velocity in the D- and E-regions, and the electron and ion temperatures in the F-region, the detachment rate of electrons from negative ions in the lower ionosphere (Bakhmet'eva et al. 1998, 2005a,b; Belikovich et al. 1975, 1993, 1996; Kagan et al. 2002). The knowledge of the ionospheric parameters is often essential for a correct interpretation of the results of radio astronomical observations.

Artificial periodic irregularities of the ionospheric plasma for the first time were detected in 1975 using the first RRI heating facility at the Zimenki Laboratory (geographical coordinates $56.14^{\circ} \mathrm{N} ; 44.38^{\circ} \mathrm{E}$, see Belikovich et al. 1975). Now the SURA heating facility $\left(56.13^{\circ} \mathrm{N} ; 46.10^{\circ} \mathrm{E}\right)$ for observations of APIs is used. APIs are formed in the field of a high-power standing radio wave resulting from interference of the wave incident on the ionosphere and the wave reflected from it. The API formation and their sounding by probe radio waves are shown schematically in Figure 1.

The standing-wave field gives rise to a structure with spatial period equal to one-half of the wavelength of a high-power radio wave. In the lower ionosphere, the formation of APIs is determined by heating effects, while in the F-region it is stipulated by the ponder motive force effect. The API formation theory and the 
results of experimental studies of the ionosphere by this method are presented in Belikovich et al. (2002).

The understanding of the API formation processes at different altitudes has led to development of methods for determining a number of main parameters of the ionospheric plasma and the atmosphere. All of them are based on the API creation and detection of the artificial periodic irregularities by probing radio waves, on the reception of signals scattered from irregularities, and on the measurement of their altitude and time dependences. The condition of resonant scattering (Bragg condition) in the case of backscattering is the equality

$$
\lambda_{1}=\lambda_{2},
$$

where $\lambda_{1}$ is the wavelength of the heating radio wave in the plasma and $\lambda_{2}$ is the radar wavelength. This condition is satisfied in the two cases, which determine two possible methods for observation and detection of APIs. The first method, which was used in most experiments, is based on the API creation and their detection by waves of the identical frequency and polarization. In this case, the condition of API detection is the equality

$$
f_{1}=f_{2},
$$

where $f_{1}$ and $f_{2}$ are the frequencies of the powerful heating and probing waves, respectively.

The second method of API creation and detection requires the use of radio waves of different frequencies and different polarizations. In this case, the condition of API detection is the relationship

$$
f_{1} n_{1}^{0, x}=f_{2} n_{2}^{x, 0}
$$

where $n_{1,2}^{0}$ and $n_{1,2}^{x}$ are refractive indexes of the ordinary $(\mathrm{O})$ and extraordinary (X) modes at the corresponding frequencies. This method of the scattered signal detection allows to determine the electron number density.

The investigations of the ionosphere during recent 15 years have used the API technique with equality of the power and probing wave frequencies with extraordinary polarization. The waves with extraordinary polarization usually are used for generation of APIs, because such waves do not excite ionospheric plasma instabilities and, therefore, they experience lower losses in the reflection from the ionospheric F-region (Belikovich et al. 2002). As a rule, the high-power wave is radiated at frequencies $f=4-6 \mathrm{MHz}$.

The measurements by the method of the resonance scattering from APIs usually are performed in the following sequence. In each measurement cycle, the heating transmitter operates for several seconds in the continuous mode, emitting an X-mode wave and creating APIs. Then the SURA facility is switched to the pulsed mode and it emits short pulses of the same frequency and polarization and having a repetition rate of $50 \mathrm{~Hz}$. After SURA facility is switched off, APIs gradually disappear. The scattered signals are received after each sounding pulse in the 50-400 km altitude range, and their sine and cosine components are recorded. The pulsed sounding continues for several seconds during the relaxation of API. This cycle is repeated several times per minute. Some examples of the altitude-time dependence of amplitudes and relaxation times of the signal, scattered by API, are presented in the Figure 2. 


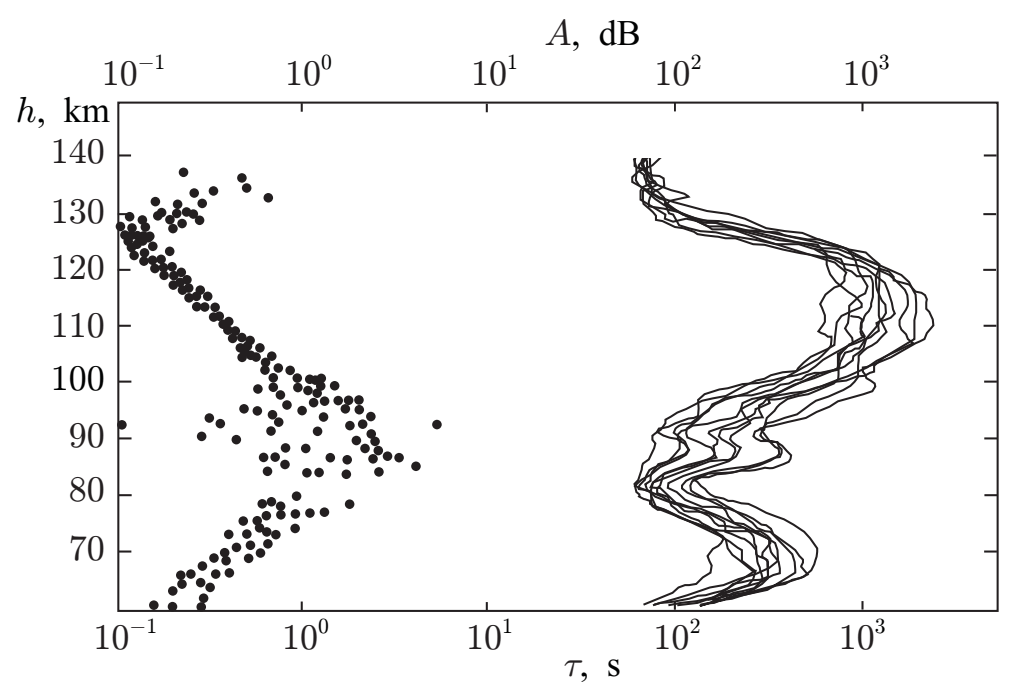

Fig. 2. Examples of the altitude dependences of the amplitude $A$ (right panel) and the relaxation time $\tau$ (left panel) of the API scattered signal in the lower ionosphere measured during one hour on 2010-09-18.

The analysis of the altitude-time dependences of the amplitude and the phase of the API scattered signal makes possible to get information on a large number of ionospheric parameters. The researchers of the Radiophysical Research Institute (Nizhny Novgorod, Russia) have developed the methods for determining main characteristics of the ionospheric plasma with the use of APIs, which include (Belikovich et al. 2002): (i) the altitude distribution of the electron number density, including the interlayer E-F valley (altitudes $60-250 \mathrm{~km}$ ); (ii) the temperature and density of the atmosphere at the altitudes of E-region (100-130 km); (iii) the velocity of a vertical motion in the D and E regions $(60-130 \mathrm{~km})$; (iv) the typical turbulent velocity near the turbopause and its altitude $(90-110 \mathrm{~km}) ;(\mathrm{v})$ the relative number density of negative ions of oxygen and concentrations of atomic oxygen and excited molecular oxygen in the ${ }^{1} \Delta_{g}$ state in the D-region $(60-90 \mathrm{~km})$; (vi) the ion composition of the sporadic E-layer $(85-130 \mathrm{~km})$; (vii) the electron and ion temperatures in the F-region (200-300 km).

Moreover, the method based on the resonant scattering of signals from APIs permits (i) to determine reliably parameters of the internal gravity waves and their spectral characteristics, (ii) to study the inhomogeneous structure of the lower ionosphere, including stratification of the regular E-layer, (iii) to detect weak sporadic layers of ionization, which are inaccessible for detection by standard ionosonde, and additional layers of the electron density profiles from the lower part of the D-region to the altitude of the F-layer maximum, and (iv) to explore the features of the sunrise and sunset phenomena in the D-region.

Now the greatest attention is given to investigation of the D-region and the sporadic E-layer $\left(E_{s}\right)$, the turbulent phenomena and the atmospheric waves at the lower ionosphere altitudes (Bakhmet'eva et al. 1999, 2005a,b; Kagan et al. 2002).

It was shown that the API relaxation time $\tau$ in the E-region is defined by ambipolar diffusion process (Belikovich et al. 2002). In this case, $\tau$ is defined by 
the following formula:

$$
\tau=\frac{1}{K^{2} D}=\frac{M_{i} \nu_{i m}}{\kappa\left(T_{e 0}+T_{i 0}\right) K^{2}}
$$

where $\kappa$ is the Boltzmann constant, $\frac{4 \pi}{\lambda}$ is a wave number of the standing wave, $\lambda=\frac{\lambda_{0}}{n}$ is the wavelength in the medium, $D_{a}$ is the ambipolar diffusion coefficient, $M$ is the molecular mass of positive ions, $T_{e 0}$ and $T_{i 0}$ are background (undisturbed) electron and ion temperatures, $\nu_{\mathrm{im}}$ is the ion and molecule collision frequency. The relaxation time is defined as a decrease of the amplitude of the scattered signal in $e$ times. The primary data processing involves calculation of the signal phase $\phi$ and amplitude $A$ at each altitude for each heating cycle and approximating their time dependences by linear functions of the form $\ln A(t)=\ln A_{0}-\frac{t}{\tau}$ and $\phi(t)=\phi_{0}+\frac{4 \pi V t}{\lambda}$, where $\tau$ characterizes the API relaxation time after switching off the heating transmitter, $V$ is the vertical velocity of plasma, $\lambda$ is the power wavelength. The vertical plasma velocity is defined by the change of the phase of the scattered signal. The temperature and density of the neutral components can be derived from the altitude dependence of the relaxation time (Belikovich et al. 1993, 1996).

\section{STUDIES OF THE SPORADIC E-LAYER}

In recent years, the method of radio-wave scattering by APIs has extensively been used for a study of the irregular structure of the ionosphere, including the sporadic ionization. The sporadic ionization layers (named sporadic E-layers or $\left.E_{\mathrm{s}}\right)$ play a crucial role in generation of small-scale irregularities and in providing reliability of radio communication and navigation. Within the framework of the concept that the mid-latitude sporadic $E_{\mathrm{s}}$-layer is formed by long-living metallic ions (Whitehead 1989; Mathews 1998), we have proposed the method for determining the molecular mass of these ions, i.e., their type and concentration, using the altitude dependence of the API relaxation time (Bakhmet'eva et al. 1999, 2005; Belikovich et al. 2002; Kagan et al. 2002). At the altitudes where the influence of the atmospheric turbulence can be neglected (above the turbopause), the relaxation of irregularities in the E-region occurs due to the ambipolar diffusion effect. In this case, in the absence of the $E_{\mathrm{s}}$-layer, the altitude dependence of the API relaxation time $\tau(h)$ is approximately exponential. The turbulence effect appears as a decrease in the relaxation time with respect to the time of the diffusion spreading of APIs.

The influence of sporadic ionization on the scattered signal is evidenced as a local increase in its amplitude $A$, which sometimes rises by $30-40 \mathrm{~dB}$ at the altitudes of the $E_{\mathrm{s}}$-layer (Bakhmet'eva et al. 1999, 2005a,b). The relaxation time of the signal in the presence of the $E_{s}$-layer also increases. It was mentioned above that without the $E_{s}$-layer, the altitude dependence of the API relaxation time $\tau(h)$ is exponential. However, if the $E_{\mathrm{s}}$-layer at some altitude exists, then in the dependence $\tau(h)$ at the altitude of the maximum of sporadic ionization we observe the local maxima of the API relaxation time, in which $\tau$ sometimes increases by several times. The appearance of such maxima of $\tau(h)$ dependence is due to the two factors. The first one is an increase in the electron number density in the $E_{\mathrm{s}}$-layer compared with the background density of the E-region, which leads to a decrease in the refractive index $n$, an increase in the wavelength in the layer, and, respectively, 
an increase in the time of diffusion spreading of irregularities. Another factor is the presence of long-lived metallic ions at these altitudes. Gathering of these ions into thin layers leads to the formation of the $E_{\mathrm{s}}$-layer at mid-latitudes, in accordance with the wind-shear theory (Whitehead 1989). The diffusion relaxation time of APIs is proportional to the molecular mass of the dominant ions, see Eq. (4). It is the way to estimate masses of the predominant metallic ions. Based on the results of spectrometric measurements (see, e.g., Grebovsky et al. 1998; Gerding et al. 2001), the ions $\mathrm{Fe}^{+}, \mathrm{Mg}^{+}, \mathrm{Ca}^{+}, \mathrm{Al}^{+}, \mathrm{Na}^{+}, \mathrm{Si}^{+}$and others in the sporadic Elayers have been detected. Eq. (4) shows that the variations of the API relaxation time are most influenced by the heavier ions, such as the ions of iron with atomic mass $M=56$, which is almost a factor of two larger than the average molecular mass of the atmospheric ions $\mathrm{NO}^{+}(M=30)$ and $\mathrm{O}_{2}^{+}(M=32)$, which are predominant at the altitudes of the E-region, or calcium ions $(M=40)$. Since, according to the measurement data, in the $E_{\mathrm{s}}$-layer heavy ions can compound up to $80 \%$ of the total number of metallic ions, a notable increase in $\tau$ at the sporadic ionization altitude can be expected. If the $N(h)$ profile of the electron density is known, then it is possible to estimate molecular masses of metallic and atmospheric ions, respectively (Bakhmet'eva et al. 2005a,b; Belikovich et al. 2002). Thus, using the experimental relaxation-time ratio $\frac{\tau E_{\mathrm{s}}}{\tau}$, one can estimate the relative molecular mass of metallic ions. According to the measurements on 2001-06-15, the values $M=39$ and 57, close to the masses of ions $\mathrm{Ca}^{+}$and $\mathrm{Fe}^{+}$. The equations are derived, allowing to calculate the concentration of molecular atmospheric and atomic metallic ions in the sporadic E-layer (Bakhmet'eva et al. 1999).

\section{A TWO-FREQUENCY API TECHNIQUE}

For measuring of the $N(h)$ profiles we propose a new method of the formation of APIs with two different space scales. The electron density $N$ is determined from the ratio of the relaxation times of the signals back-scattered by the API at two frequencies. The API are formed by the powerful radio waves at the frequencies $f_{1}$ $=5.65 \mathrm{MHz}$ and $f_{2}=4.7 \mathrm{MHz}$. API relaxation times $\tau_{1,2}$ at the E-region altitudes are formed by an ambipolar diffusion: $\tau_{1,2}=\left(K_{1,2}^{2} D_{\alpha}\right)^{-1}$, where $K_{1,2}=\frac{4 \pi n_{1,2}}{\lambda_{0(1,2)}}$, and $\lambda_{0(1,2)}$ are the lengths of the power waves in vacuum, $n_{1,2}$ are their refractive indexes. Therefore, the ratio of the relaxation times at the same altitude depends on the ratio of the refractive indexes for heating waves with different wavelengths and their frequencies, and this makes possible to determine the electron density. An example of the measured $N(h)$ profile is presented in Figure 3.

The two-frequency measurements show different types of the $N(h)$-profiles. First, there are $N(h)$ profiles close to the models of the regular E-region. Between midday and evening the average electron density decreases in accordance with the atmospheric ionization by solar radiation, i.e., $N$ depends on the solar zenith angle. Second, the altitude dependencies of the electron density, including the sporadic layers, can be observed. Third, sometimes the stratification of the Eregion at the altitude of its maximum is observed. At last, wave-like changes of $N$ with the altitude are also observed frequently. It was showed that those effects could be explained by the internal gravity wave propagation and instabilities of the environment within the investigated region. Also we have observed oscillations of the electron density with a period of about 30 minutes at the maximum altitude of 


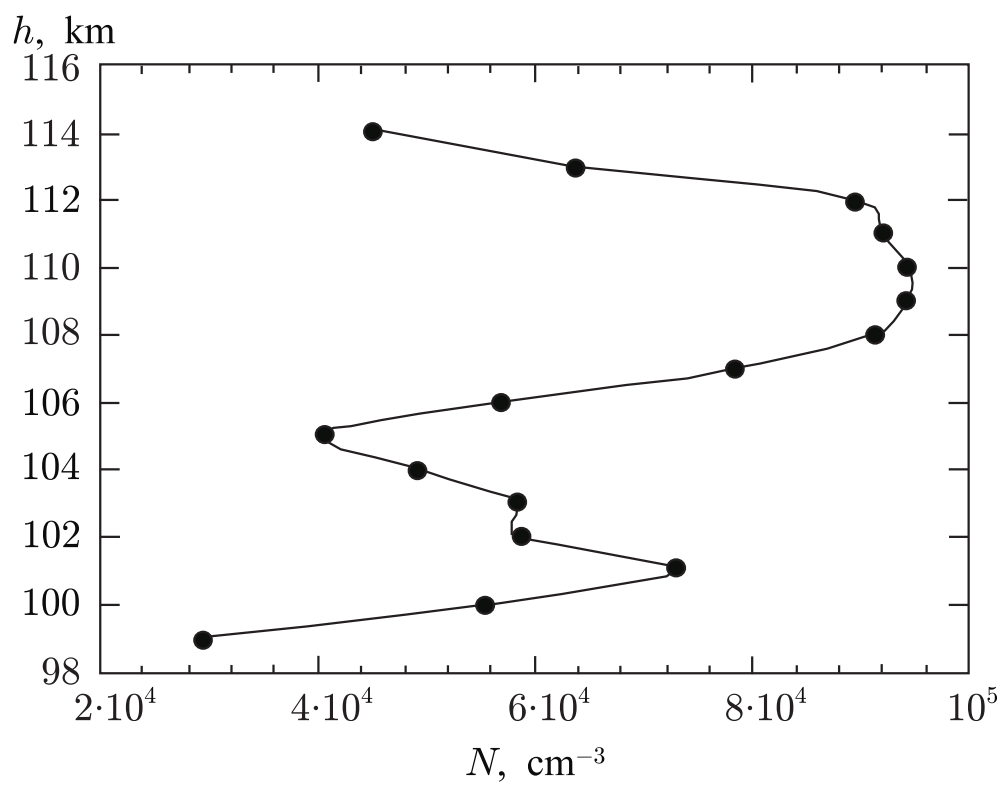

Fig. 3. The altitude profile of the electron concentration $N(h)$ obtained for the time 16:29 by two-frequency API measurements on 2006-10-04. The local maximum of the $N(h)$-profile at an altitude of $101 \mathrm{~km}$ corresponds to the sporadic E-layer and $N(h)$ maximum at an altitude of $110 \mathrm{~km}$ shows the maximum electron density of E-region.

the E-region. They have existed quite long, some hours. Their occurrence could be connected with the steady propagation of the internal gravity waves (Bakhmet'eva et al. 2010; Tolmacheva et al. 2011).

\section{WAVE DISTURBANCES}

The wave motions at the altitudes of the lower ionosphere are distinctly demonstrated by temporal-space variations of the temperature, density and other parameters. Two types of the altitude profiles of $T(h)$ were obtained. Profiles of the first type are smooth. On profiles of the second type wavy variations with the characteristic altitude scale of $4-6 \mathrm{~km}$ are observed. We consider that these variations are caused by the internal gravity waves (IGWs). The height interval of the observations is limited by the turbulence in the bottom region and by the instrumental parameters at the top region. At present we can identify correctly only IGWs with a vertical wavelength of $\lambda_{z}<8-9 \mathrm{~km}$. The propagation of longer waves can be evidenced by temporary variations of the temperature and other parameters of the ionosphere (Bakhmet'eva et al. 2010; Belikovich et al. 2002). In Figure 4 an example of the temperature variations with altitude is presented.

For example, in the experiment carried out on 2007 September 24 and 2010 September 18 the periods of variations at 20-30, 40-50, 80-90 minutes, and up to 2-4 hours were observed. We consider that the atmospheric conditions are undisturbed when the smooth height dependences $T(z)$ of the first type take place. They can exist during some hours. Nevertheless, if there is a great negative temperature gradient, the altitude-temporal variations of the neutral temperature and density 


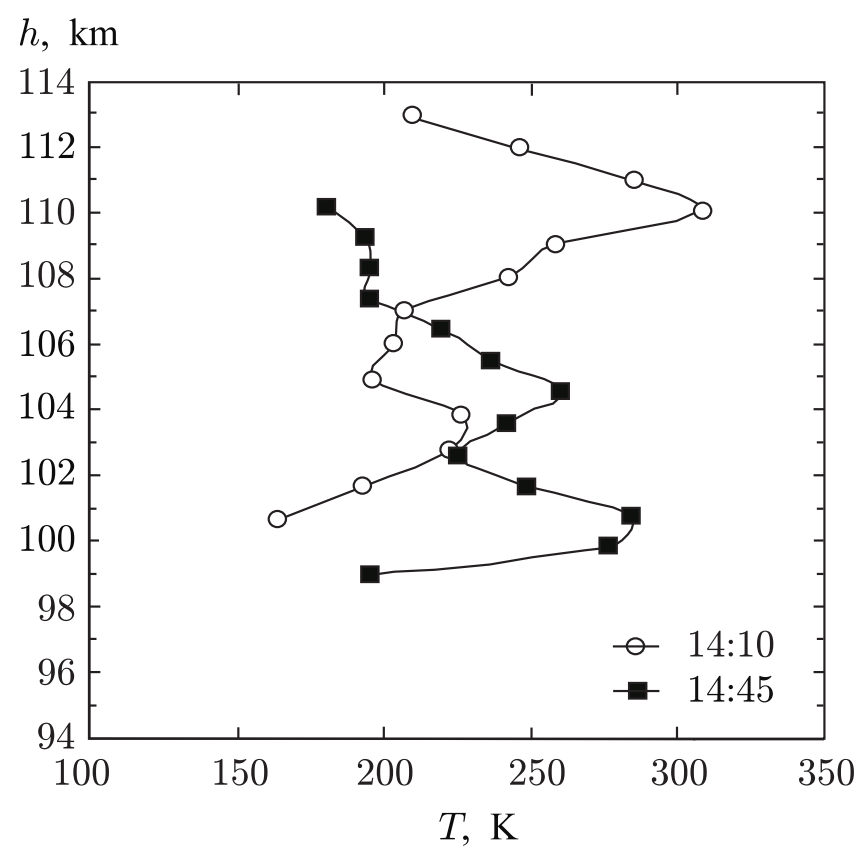

Fig. 4. The temperature vs. altitude profiles of neutral component of the ionosphere for two sessions of measurements on 2007-09-24.

appear, and they can exist for a long time. Such phenomena could be connected with the evolution of hydrodynamic instability and with the generation of internal gravity waves (Bakhmet'eva et al. 2010).

\section{CONCLUSIONS}

The API technique allows to perform systematic studies of the plasma parameters in the lower ionosphere. APIs were observed after artificial heating at Arecibo (Fejer et al. 1984), EISCAT at Tromso (Rietveld et al. 1996; Rietveld \& Goncharov 1998) and HIPAS in Alaska (Djuth et al. 1997). The ionosphere investigation method with the API technique continues to develop. The new method for determining of electron density from the API relaxation time measurements at two different frequencies was recently proposed. The method opens the way to a more precise measurement of the altitude profile of electron density in the E-region, as well as the temperature and density of the neutral atmosphere at the altitudes of $90-115 \mathrm{~km}$. This also opens the possibility for determining the ambipolar diffusion coefficient and the turbulent motion velocity, independently. The API method also exceeds other methods of the remote ionospheric sounding by the information value. The plasma parameter measurements based at the API technique affords a high temporal resolution, which favorably distinguishes them, e.g., from the method of the incoherent scattering, and may rival economical rocket researches. The method is environmentally clean, because it does not introduce additional impurities into the atmosphere. In the API measurements, the heating of the electron plasma component is insignificant and does not disturb the thermal balance of the neutral atmosphere. It is important that all methods of plasma 
diagnostics based on the API technique can be easily combined into a common complex technique for research and monitoring of the ionosphere in a wide range of altitudes, from D- to F-region.

ACKNOWLEDGMENTS. The authors are thankful the Russian Foundation of Basic Research for financial support of the investigation under Grant No. 1102-97028.

\section{REFERENCES}

Bakhmet'eva N. V., Belikovich V. V., Benediktov E. A. et al. 1997, Radiophysics and Quantum Electronics, 40, 196

Bakhmet'eva N. V., Belikovich V. V., Benediktov E. A. et al. 1998, Radio Sci., 33,583

Bakhmet'eva N. V., Belikovich V. V., Ignat'ev Yu. A., Ponyatov A. A. 1999, Radiophysics and Quantum Electronics, 42, 22

Bakhmet'eva N. V., Belikovich V. V., Kagan L. M., Ponyatov A. A. 2005a, Radiophysics and Quantum Electronics, 48, 14

Bakhmet'eva N. V., Belikovich V. V., Kagan L. M. et al. 2005b, Radiophysics and Quantum Electronics, 48, 673

Bakhmet'eva N. V., Grigor'ev G. I., Tolmacheva A. V. 2010, Radiophysics and Quantum Electronics, 53, 623

Belikovich V. V., Benediktov E. A., Getmantsev G. G., Komrakov G. P., Ignat'ev Yu. A. 1975, JETP Letters, 22, 497

Belikovich V. V., Benediktov E. A., Tolmacheva A. V. 1993, Geomagnetism and Aeronomy (English translation), 34, 115

Belikovich V. V., Benediktov E. A., Goncharov N. P., Tolmacheva A. V. 1996, Geomagnetism and Aeronomy (English translation), 35, 490

Belikovich V. V., Benediktov E. A., Tolmacheva A. V., Bakhmet'eva N. V. 2002. Ionospheric Research by Means of Artificial Periodic Irregularities, Copernicus GmbH, 2002, Katlenburg-Lindau, 160 pp.

Djuth F. T., Groves K. M., Elder J. H. et al. 1997, J. Geophys. Res., 102, 24023

Fejer J. A., Djuth F. T., Gonzales C. A. 1984, J. Geophys. Res., 89, 9145

Grebovsky J. M., Goldberg R. A., Pesnell W. D. 1998, J. Atmos. Sol.-Terr. Phys., 60, 607

Gerding M., Alpers M., Hoffner J., von Zahn U. 2001, Ann. Geophys., 19, 47

Mathews J. D. 1998, J. Atmos. Sol.-Terr. Phys., 60, 413

Kagan L. M., Bakhmet'eva N. V., Belikovich V. V. et al. 2002. Radio Sci., 37, 1106

Rietveld M. T., Turunen E., Matveinen H. et al. 1996. Ann. Geophys., 14, 1437

Rietveld M. T., Goncharov N. P. 1998, Adv. Space Res., 21, 693

Tolmacheva A. V., Bakhmet'eva N. V., Vyakhirev V. D. et al. 2011, Radiophysics and Quantum Electronics, 54, 365

Whitehead J. D. 1989, J. Atmos. Terr. Phys., 51, 401 